\title{
Isolation and characterization of a carotenoid oxygenase gene from Chlorella zofingiensis (Chlorophyta)
}

Jun-Chao Huang · Yan Wang · Gerhard Sandmann · Feng Chen

J.C. Huang $\cdot$ F. Chen $(\bowtie)$

Department of Botany, the University of Hong Kong,

Pokfulam Road, Hong Kong, China

e-mail: sfchen@hkusua.hku.hk

Tel.: +852-2299-0309

Fax: $+852-2299-0311$

Y. Wang $\cdot$ F. Chen

South China Sea Institute of Oceanology, Chinese Academy of Sciences, Guangzhou, China

G. Sandmann

Botanical Institute, Goethe University Frankfurt, P.O. Box 111932, D-60054 Frankfurt, Germanny 


\begin{abstract}
The green alga Chlorella zofingiensis produces large amounts of the valuable ketocarotenoid astaxanthin under dark-heterotrophic growth conditions, making it potentially employable for commercial production of astaxanthin as feed additives, colorants, and health products. Here, we report the identification and characterization of a carotenoid oxygenase (CRTO) gene that is directly involved in the biosynthesis of ketocarotenoids in C. zofingiensis. The open reading frame of the crtO gene, which is interrupted by three introns of 243, 318, and $351 \mathrm{bp}$, respectively, encodes a polypeptide of 312 amino acid residues. Only one crtO gene was detected in the genome of $C$. zofingiensis. Furthermore, the expression of the crtO gene was found up-regulated upon glucose treatment. Functional complementation in Escherichia coli showed that the coding protein of the crtO gene not only exhibits normal CRTO activity by converting $\beta$-carotene to canthaxanthin via echinenone, but also displays a high enzymatic activity of converting zeaxanthin to astaxanthin via adonixanthin. Based on the bifunctional CRTO, a predicted pathway for astaxanthin biosynthesis in C. zofingiensis is described and the CRTO is termed as carotenoid $4,4^{\prime}-\beta$-ionone ring oxygenase.
\end{abstract}

Key words: Astaxanthin; carotenoid oxygenase; Chlorella zofingiensis 


\section{Introduction}

Carotenoids are important natural pigments that may be produced by many microorganisms and plants (Johnson and Schroeder 1995; Lee and Schmidt-Dannert 2002). The ketocarotenoid astaxanthin has recently attracted much attention due to its strong antioxidant properties that make it having diverse biological functions such as protection against UV-light photooxidation, inflammation, cancer, and age-related diseases (Lorenz and Cysewski 2000; Guerin et al. 2003). Astaxanthin is commonly found in marine animals, e.g. crustaceans, shellfish, and salmonoids (Lorenz and Cysewski 2000). These animals do not possess the ability to synthesize the carotenoid pigment de novo. Instead they acquire the pigment from their diet. Astaxathin has been exploited as a feed supplement for fish and shellfish.

The biosynthesis of astaxanthin is limited to some microorganisms, e.g. the yeast Xanthophyllomyces dendrorhous (formerly Phaffia rhodozyma), the freshwater alga Haemoatococcus pluvialis and the marine bacteria Agrobacterium aurantiacum (Johnson and An 1991; Lorenz and Cysewski 2000). The final steps in astaxanthin formation are ketolation and hydroxylation of $\beta$-carotene. In all studied organisms except $X$. dendrorhous the two modification reactions of $\beta$-carotene are independent. Two different pathways of astaxanthin biosynthesis were proposed: one starting from the oxidation of $\beta$-carotene producing intermediates of echinenone, canthaxanthin and adonirubin, the other from the hydroxylation of $\beta$-carotene with $\beta$-cryptoxanthin, zeaxanthin and adonixanthin as intermediates (Fig. 1) (Sandmann, 1994; Cunningham and Gantt, 1998; Margalith, 1999). The first pathway has been demonstrated by functional analysis of the CrtW/BKT type diketolases from the marine bacteria A. aurantiacum and green alga $H$. pluvialis, in that the ketolases only accept $\beta$-carotene as 
substrate (Lotan and Hirschberg 1995; Misawa et al. 1995; Breitenbach et al. 1996; Linden 1999). No information related to the second pathway has yet been available.

Although $H$. pluvialis is a potential commercial source of natural astaxanthin, the slow growth rate and relatively low growth temperature of this alga greatly hinder its application on a large-scale (Margalith 1999; Guerin et al. 2003). The chlorophycean microalga Chlorella zofingiensi was reported to accumulate high amounts of astaxanthin and to reach very high values of biomass when grown photoautotrophically in batch culture (Bar et al. 1995; Del Campo et al. 2000; Orosa et al. 2000). Moreover, this alga showed excellent growth and high yield of astaxanthin on glucose-supplemented media in batch culture in the dark (Ip and Chen 2005), suggesting that the alga might be potentially employed for commercial production of astaxanthin on a large-scale. However C. zofingiensis contains much less astaxanthin than $H$. pluvialis within the cell. Information of astaxanthin biosynthesis on C. zofingiensis is limited. Compared with H. pluvialis, C. zofingiensis contains a relatively high amount of canthaxanthin (about $30 \%$ of total ketocarotenoids), indicating that canthaxanthin might be the end product of the oxygenation of $\beta$-carotene. Thus, in $C$. zofingiensis astaxanthin might be synthesized via a different pathway from that in $H$. pluvialis: the oxygenation of zeaxanthin rather than the hydroxylation of canthaxanthin. This hypothesis has so far remained elusive. Based on the prediction, C. zofingiensis may consist of either two oxygenases (one for specifically converting $\beta$-carotene to canthaxanthin and the other for zeaxanthin to astaxanthin) or only one oxygenase with bifunctional activities of converting $\beta$-carotene to canthaxanthin as well as zeaxanthin to astaxanthin. The identification of the carotenoid oxygenase gene from C. zofingiensis is the key to revealing the astaxanthin biosynthesis in the alga. 
The aim of this study is to isolate the carotenoid oxygenase gene from the important astaxanthin-producing green alga C. zofingiensis, characterize its function, and reveal its expression under conditions for ketocarotenoid accumulation. The putative pathway of astaxanthin biosynthesis in this alga is discussed.

\section{Materials and methods}

Strains and culture conditions

The green microalga, C. zofingiensis (ATCC30412) was purchased from American Type Culture Collection (ATCC, Rockville, USA). The alga was maintained and cultured in a basal medium consisting (per liter) of $2 \mathrm{~g}$ yeast extract, $0.4 \mathrm{~g}$ L-asparagine, $1.2 \mathrm{~g} \mathrm{CH}_{3} \mathrm{COONa}, 0.2 \mathrm{~g}$ $\mathrm{MgC}_{12} 6 \mathrm{H}_{2} \mathrm{O}, 0.02 \mathrm{~g} \mathrm{CaCl}_{2} 2 \mathrm{H}_{2} \mathrm{O}$ and $0.01 \mathrm{~g} \mathrm{FeSO}_{4} 7 \mathrm{H}_{2} \mathrm{O}$ (pH 6.8) (Kobayashi et al., 1993). $C$. zofingiensis was subcultured in the basal medium at $25{ }^{\circ} \mathrm{C}$ under conditions of continuous

illumination $\left(30 \mu \mathrm{mol} \mathrm{m} \mathrm{m}^{-2}\right)$ for 5 days. To induce astaxanthin biosynthesis, glucose was added to the above culture at a final concentration of $20 \mathrm{~g} \mathrm{l}^{-1}$. Samples $(15 \mathrm{ml})$ were collected at intervals (6h, 12h, 24h, 36h, and 48h) and harvested by centrifugation.

Escherichia coli JM109 was used as a host for functional characterization of the bkt gene product from C. zofingiensis.

Genomic DNA and RNA isolation 
DNA and RNA techniques were followed according to the standard methods described in Sambrook et al. (1989). DNA was extracted using a modified CTAB method (Stewart and Via, 1993). RNA was isolated from aliquots of about $10^{8}$ cells harvested at different stages of astaxanthin accumulation using the TRI reagent (Molecular Research Center) according to the manufacturer's instructions. The concentration of total DNA and RNA was determined spectrophotometrically at $260 \mathrm{~nm}$.

Cloning of $b k t$ cDNA and its correspondent gene

Degenerate primers were designed for the amplification of a partial bkt cDNA from $C$. zofingiensis. The primers were derived from the conserved nucleotide and amino acid sequences reported for the bkt genes from H. pluvialis, Agrobacterium aurantiacum and Alcaligenes PC-1 (Misawa et al., 1995; Kajiwara et al., 1995; Lotan and Hirschberg 1995). Primers dbktfl (forward: 5'-GTTYYTNTAYACNGGNYTNTTYAT) and dbktr1 (reverse 5'CARRTANGTNCCRAARTARAA) were derived from motifs FLYTGLFI and FYFGTYLP. Primers dbktf2 (forward: 5'-CACIACICAYGAYGCIATGCAYGG) and dbktr2 (reverse: 5'CCIYKRTGRAARTCIGGRTC) were derived from TTHDAMHG and DPDFHKG. One step RT-PCR with primers dbktf1 and dbktr1 was performed to amplify a portion of a bkt cDNA. Total RNA (100 ng) was from cells induced with 2\% glucose for $24 \mathrm{~h}$. RT-PCR conditions were: $45{ }^{\circ} \mathrm{C}$ for 15 min for cDNA synthesis, followed with PCR amplification $\left(35\right.$ cycles of $94{ }^{\circ} \mathrm{C}$ for $20 \mathrm{~s}, 42^{\circ} \mathrm{C}$ for $20 \mathrm{~s}, 72{ }^{\circ} \mathrm{C}$ for $40 \mathrm{~s}$ ). A nested PCR (with primers dbktf2 and dbktr2) was performed using the same PCR cycles. The nested PCR product was cloned into pTZ57R/T vector (MBI Fermentas) according to the manufacturer's instructions. Positive clones were 
selected and sequenced in both directions using an automated ABI3100 sequencer. The sequence of putative insert was used for designing specific primers for 5' and $3^{\prime}$ RACE (5'CGACGGTTCCTGATGGCAATAGT and 5'-CAGCATGTCATAGTCAAACCAGGC for 5' RACE; 5'-GCCTGGTTTGACTATGACATGCT and 5'-GAAGCACTGGGAGCATCACAACT for $\left.3^{\prime} \mathrm{RACE}\right)$. RACE was performed using a 5'/3' RACE kit according to the manufacturer's instructions (Roche molecular biochemicals, Germany).The RACE products were gel purified and sequenced. One pair of specific primers (forward: 5'-TTCCTGGGCCGTGCTGTATT; reverse: 5'-AACCTTGATTAAGTTACATGCT) was designed from the sequences of the 5' and 3' RACE fragments to amplify a full length $b k t$ cDNA and its correspondent gene.

Southern blot

Eight mg of genomic DNA was digested with KpnI, BamHI + EcoRI and PstI + XbaI, five restriction enzymes without a recognition sites in the probed regions of the ketolase genes. The digested DNA was separated with a $0.8 \%$ agarose gel, transferred to a positively charged nylon membrane (Boehringer Mannheim/Roche), and hybridized with Dig-labeled DNA probes in the presence of $50 \%\left(\mathrm{v} \mathrm{v}^{-1}\right)$ formamide at $47^{\circ} \mathrm{C}$ for $16 \mathrm{~h}$. DNA probe was prepared by amplifying a 1090 bp fragment of bkt gene with a pair of specific primers (forward: 5'ATGGCTGCTGGCAAATCA and reverse: 5'-GCCGCCAGAAAGACGCATA) and a plasmid containing the bkt gene template ( 30 cycles of $94{ }^{\circ} \mathrm{C}$ for $20 \mathrm{~s}, 60^{\circ} \mathrm{C}$ for $20 \mathrm{~s}, 72{ }^{\circ} \mathrm{C}$ for $1 \mathrm{~min}$ ). Probe labeling and hybridization were carried out according to the instructions in the DIG Nonradioative Nucleic Acid Labeling and Detection System (Boehringer Mannheim/Roche). 
After hybridization, the membrane was washed twice with $0.1 \times$ SSC containing $0.1 \%$ SDS at 68 ${ }^{\circ} \mathrm{C}$ for $15 \mathrm{~min}$.

Functional analysis of $b k t$ cDNA

The open reading frame of bkt was PCR amplified and cloned into the vector pBluescript II KS as an in-frame fusion to the lacZ gene resulting in plasmid pCzb. E. coli strain JM109 was used as a host for complementation experiments by co-transformation of $\mathrm{pCzb}$ with plasmids either pACCAR $16 \Delta$ crtX or pACCAR25 $\Delta$ crtX that harbor the carotenoid biosynthesis genes for producing $\beta$-carotene or zeaxanthin, respectively (Misawa et al., 1995).

Pigment analysis

Carotenoids were extracted and analyzed according to McCarthy et al. (2004). E. coli cells were collected by centrifugation and freeze-dried. Extraction was carried out with a mixture of dichloromethane and methanol $\left(25: 75, \mathrm{vv}^{-1}\right)$ until the cell debris was almost colorless. The combined extracts were evaporated to dryness and separated on a $5 \mu \mathrm{m}$ ODS2 $4.6 \times 250 \mathrm{~mm}$ analytical column (Waters Spherisorb ${ }^{\circledR}$ ) with a Waters high-performance liquid chromatograph. Individual carotenoids were identified by absorption spectra and their typical retention times compared to standard samples of pure carotenoids.

RT-PCR assay 
Total RNA $(1 \mu \mathrm{g})$ extracted from samples treated with glucose for 6 to $48 \mathrm{~h}$ (see 2.1.) was reverse transcribed to cDNA with SuperScript ${ }^{\mathrm{TM}}$ III First-Strand Synthesis System for RT-PCR (Invitrogen) primed with oligo-dT according to the manufacturer's instructions. PCR amplification was done using bkt forward 5'-ATGGCTGCTGGCAAATCA and reverse 5'GCCGCCAGAAAGACGCATA primers. C. zofingiensis actin primers (forward: 5'TGCCGAGCGTGAAATTGTGAGG; reverse: 5'-CGTGAATGCCAGCAGCCTCCA) were used to monitor equality of templates and loading. Amplification of the cDNA was done by conventional PCR $\left(94{ }^{\circ} \mathrm{C}\right.$ for $2 \mathrm{~min}$ followed by 24 cycles (for bkt gene) or 22 cycles (for actin gene) of $94{ }^{\circ} \mathrm{C}$ for $15 \mathrm{~s}, 59{ }^{\circ} \mathrm{C}$ for $15 \mathrm{~s}, 72{ }^{\circ} \mathrm{C}$ for $30 \mathrm{~s}$ ). PCR products were separated on $1.5 \%$ agarose gels and stained with ethidium bromide for photography (Biorad).

\section{Results}

Cloning and characterization of the carotenoid oxygenase gene (crtO) from C. zofingiensis

Two pairs of degenerate primers were designed from the conserved motifs presenting among all known diketolases. RT-PCR with dbktfl and dbktr1 (see material) could not generate clonable fragments (data not shown). However a nested PCR with primers dbktf2 and dbktr2 generated a predicted $180 \mathrm{bp}$ fragment (Fig. 2. lane 1). BLAST analysis showed that the sequence of the fragment was derived from a putative ketolase. With the sequence information, specific primers were designed for 5' and 3' RACE of the related gene. 5' RACE generated a $600 \mathrm{bp}$ fragment and 3' RACE produced a $1400 \mathrm{bp}$ fragment (Fig. 2). They were revealed by sequence as the 5' and 3' regions of a ketolase gene. RT-PCR with a pair of primers annealing to the most ends of 
the 5' and 3' RACE fragments generated a $2 \mathrm{~kb}$ fragment, confirming the presence of the cDNA (Fig. 2. lane 4). The RT-PCR fragment was identified as a full-length $c r t O / b k t c D N A$ (Genbank accession no. AY772713). The open reading frame of this cDNA encodes a protein of 312 amino acids that shares 53\% identical sequence with the H. pluvialis BKT (Kajiwara et al., 1995). The GC content of the crtO coding region is $51.1 \%$, which is lower than that of the bkt from $H$. pluvialis (57.1\%).

To characterize the correspondent gene of the crtO cDNA, Genomic PCR was performed. A $3 \mathrm{~kb}$ fragment was generated and sequenced. Analysis of the obtained nucleotide sequence revealed that the product was the correspondent gene of the crtO cDNA (Genbank accession number AY772714). The particular crtO gene consists of four exons and three introns of 243, $318,351 \mathrm{bp}$. Intron/extron splice sites of the $\mathrm{crtO}$ gene are strongly conserved. All introns start with GT and end with CAG. The first and last base of the exons is a G, which is strongly conserved as in the case of higher plants (Brown, 1989). The sequence TGTAA, which was considered as a potential polyadenylation signal of green algae is located $13 \mathrm{bp}$ upstream of the polyadenylation site. Similar to Chlamydomonas genes, the $\mathrm{crtO}$ gene is also characterized by a long 3' noncoding region of about $1 \mathrm{~kb}$ in length.

To detect the number of $\mathrm{crtO}$ gene in the haploid genome of C. zofingiensis, genomic DNA was digested with different restriction enzymes and subjected to Southern blot analysis. Using a $1 \mathrm{~kb}$ fragment of $\mathrm{crtO}$ as a probe, the homologous fragments showed strong hybridization signals (Fig. 3.). Each of the three separate digests showed only one band, suggesting the presence of only one $\mathrm{crtO}$ gene in the haploid genome of C. zofingiensis.

Functional analysis of the $c r t O$ cDNA in E. coli 
In order to find out the enzymatic activity of the ketolase encoded by the $\operatorname{crtO}$ gene, the ORF of the gene was PCR-amplified and inserted into the vector pBluescript II KS+ as an in-frame fusion to the lacZ gene and the resulting plasmid (pCzcrtO) was co-transformed with pACCAR16 $\Delta$ crtX or pACCAR25 $\Delta$ crtX respectively into E. coli JM109. E. coli JM109 harboring plasmid pACCAR $16 \Delta$ crtX or pACCAR25 $\Delta$ crtX displays a yellow phenotype due to the accumulation of $\beta$-carotene or zeaxanthin. Transformants carrying pCzcrtO with pACCAR $16 \Delta$ crtX or pACCAR25 $\Delta$ crtX both display an orange phenotype, indicating the formation of new pigments. HPLC analysis of pigments from transformants is shown in Fig. 4. Compared with pigments extracted from E. coli harboring only the pACCAR16 $\Delta$ crtX which accumulates $\beta$-carotene (Fig. 4B), the E. coli containing pCzcrtO and pACCAR16 $\Delta$ crtX accumulated canthaxanthin (peak 1) and echinenone (peak 2) in addition to $\beta$-carotene (peak 3) (Fig. 4A). These results indicate that the $c r t O$ cDNA encodes a protein with enzymatic activity of converting $\beta$-carotene to echinenone and canthanxanthin. Thus in this aspect, the ketolase from C. zofingiensis possesses the same enzymatic function as that from $H$. pluvialis and A. aurantiacum. Interestingly, besides zeaxanthin (Fig. 4C and D peak 6), substantial amounts of astaxanthin (Fig.4C peak 4) and adonixanthin (peak 5) were identified in the pigments from $E$. coli transformants carrying the pCzcrtO and pACCAR25 $\Delta \mathrm{crtX}$. These results demonstrate that the enzyme encoded by the crtO gene from C. zofingiensis is able to convert zeaxanthin to astaxanthin via adonixanthin efficiently. Coupled with Southern blot analysis, it is clear that $C$. zofingiensis only contains one carotenoid ketolase with bifunctional activities of converting $\beta$ carotene to canthaxanthin as well as zeaxanthin to astaxanthin. 
Expression of the Chlorella crtO gene during the ketocarotenogenesis

C. zofingiensis was found to accumulate ketocarotenoids rapidly in response to high concentrations of glucose with or without light (Ip et al., 2004; Ip and Chen, 2005). To determine the extent of glucose regulation of the key ketocarotenoid biosynthesis gene, the expression of crtO upon glucose treatment was analyzed. Photoautotrophically grown cells at the exponential growth phase were treated with $2 \%$ glucose for $6 \mathrm{~h}$ to $48 \mathrm{~h}$. RT-PCR analysis showed that noneinduced green cells had only small amounts of crtO mRNA and the level of crtO mRNA remained stable in $48 \mathrm{~h}$ (Fig. 5 lane 1 and 7), while the steady-state mRNA level was found increased immediately upon the addition of $2 \%$ glucose, reaching its maximum at $24 \mathrm{~h}$ (Fig. 5. lane $2,3,4)$. The steady-state mRNA level began decreasing after $24 \mathrm{~h}$ upon onset of glucose (Fig. 5. lane 5,6).

\section{Discussion}

The carotenoid ketolases are enzymes that exclusively participate in the secondary carotenoid biosynthetic pathway to astaxanthin. This enzyme plays an essential role in stress-dependent initiation of astaxanthin biosynthesis. Three types of carotenoid ketolases have been found from different organisms (Cunnigham and Gantt, 1998). The bkt one from H. pluvialis and $c r t W$ from Agrobacterium aurantiacum and Alcaligenes PC-1 share highly homologous amino acid sequences (Lotan and Hirschberg, 1995; Kajiwara et al. 1995; Misawa et al. 1995). This type of ketolase acts symmetrically introducing two keto groups on both of the two $\beta$-ionone rings of $\beta$ carotene to generate canthaxanthin. Carotenoid biosynthesis genes were normally isolated by 
using complicated library-screening approaches (Kajiwara et al., 1995; Lotan and Hirschberg 1995; Linden 1999). In this study, we presented a simple but efficient approach for directly isolating the carotenoid oxygenase gene from C. zofingiensis. The approach should be applicable for rapidly isolating the diketolase type of genes from other ketocarotenoid-producing organisms, e.g. the astaxanthin-producing green algae (Orosa et al. 2000) and canthaxanthin-producing cyanobacteria (Steiger and Sandmann 2004).

Interestingly, besides possessing such normal enzymatic activity as converting $\beta$-carotene to canthaxanthin, the C. zofingiensis ketolase exhibits a novel enzymatic activity that can interact with the hydroxylase enzyme coded by the bacterial crtZ effectively, resulting in substantial amounts of adonixanthin and astaxanthin (Fig. 4C). In contrast, the ketolases from H. pluvialis and A. aurantiacum are very poor in the conversion of zeaxanthin to astaxanthin (Lotan and Hirschberg 1995; Breitenbach et al., 1996; Fraser et al., 1998). Recently a ketolase from the cyanobacterium Nostoc punctiforme was detected also with an efficient conversion of zeaxanthin to astaxanthin (Steiger and Sandmann, 2004). The ketolase described here is the first one that has been established with high efficient conversion of zeaxanthin to astaxanthin from eukaryote alga. Based on its function, the C. zofingiensis ketolase is termed carotenoid 4, 4'- $\beta$-ionone ring oxygenase. The formation of adonixanthin in both transforming E. coli (Fig. 4 C) and induced C. zofingiensis (data not shown) suggests that in C. zofingiensis astaxanthin is synthesized via the oxygenation of zeaxanthin (Fig. 1.). Our preliminary study revealed that C. zofingiensis accumulated zeaxanthin when the cells were treated with glucose and diphenylalamine (DPA, an inhibitor specific to ketolase enzyme) indicating that the conversion of zeaxanthin to astaxanthin was blocked due to the inhibition of the ketolase by DPA (data not shown). Because substantial canthaxanthin (about $30 \%$ of total ketocarotenoids) is accumulated in C. zofingiensis, this 
pigment may represent the end product of oxygenated $\beta$-carotene (Rise et al., 1994). Thus the carotenoid hydroxylase in this alga may not accept canthaxanthin as a substrate. The carotenoid hydroxylases from Erwinia uredovora (Breitenbach et al. 1996), A. aurantiacum (missawa et al. 1995), and H. pluvialis (Linden 1999) are able to convert canthaxanthin into astaxanthin, while the hydroxylases from cyanobacteria are not able to accept echinenone or canthaxanthin as substrates (Albrecht et al., 2001). Thus the enzymatic activities of carotenoid ketolase and hydroxylase are organism dependant and may decide the pathway of astaxanthin biosynthesis. Furthermore, their co-operation is crucial for the synthesis of substantial amounts of astaxanthin (Fraser et al. 1997; Steiger and Sandmann 2004). We predict that the astaxanthin pathway in $C$. zofingiensis occurs through the initial formation of zeaxanthin via adonixanthin, which is then converted to astaxanthin by crtO enzyme. Due to the bifunctional crtO enzyme, substantial canthaxanthin is also produced, which will accumulate because of the ineffective conversion of canthaxanthin to astaxanthin by the hydroxylase. However the yet to be isolated hydroxylase gene from C. zofingiensis prevents the confirmation of this conversion.

Chlorella species can use glucose as carbon source (Tanner, 1969; Shi et al., 1999). We found that a high concentration of glucose (up to $4 \%$ ) not only stimulated the growth of $C$. zofingiesis cells, but also enhanced the accumulation of ketocarotenoids in the alga cells (Ip et al., 2004; Ip and Chen, 2005). In contrast, $H$. pluvialis only accumulates secondary carotenoids after the cessation of cell growth (Margalith, 1999; Boussiba, 2000). Glucose triggers the transcription of mRNAs coding for such proteins as membrane transporters and glycolytic enzymes (Wolf et al., 1991; Stadler et al., 1995). Interestingly, we found that glucose also up-regulated the expression of $\mathrm{crtO}$ gene in C. zofingiensis (Fig. 5), but that how glucose triggers the expression of the $c r t O$ gene remained unknown. 
C. zofingiensis is an attractive candidate for mass production of high-value ketocarotenoids. It also represents a unique model system for studying the biosynthesis and regulation of ketocarotenoid. The cloning of the $c r t O$ gene from this alga and the illustration of its specific function provide valuable insight into the biosynthesis of astaxanthin in green microalgae. The crtO gene described here is suitable for the production of astaxanthin in such hosts that contain substantial amounts of zeaxanthin, e.g. in green algae or plants.

\section{Acknowledgements}

We are grateful to Dr K.W. Fan for technical assistance. This work was supported partially by a grant from the Research Grants Council of Hong Kong, and the Outstanding Young Researcher Award of the University of Hong Kong, the Frontier Research Grant of the SCSIO, and the Hundred-Talents scheme of Chinese Academy of Science. 


\section{References}

Albrecht M, Steiger S, Sandmann G (2001) Expression of a ketolase gene mediates the synthesis of canthaxanthin in Synechococcus leading to resistance against pigment photodegradation and UV-B sensistivity of photosynthesis. Photochem Photobiol 73:551-555

Bar E, Rise M, Vishkautsan M, Arad S (1995) Pigments and structural changes in Chlorella zofingiensis upon light and nitrogen stress. J Plant Physiol 146:527-534

Boussiba S (2000) Carotenogenesis in the green alga Haematococcus pluvialis: Cellular physiology and stress response. Physiol Plant 108:111-117

Breitenbach J. Misawa N, Kajiwara S, Sandmann G (1996) Expression in Escherichia coli and properties of the carotene ketolase from Haematococcus pluvialis. FEMS Microbiol Lett $140: 241-246$

Brown JW (1989) A catalogue of splice junction and putative branch point sequences from plant introns. Nucleic Acids Res 14:9549-9559

Cunnigham FX, Gantt E (1998) Genes and enzymes of carotenoid biosynthesis in plants. Annu Rev Plant Physiol Plant Mol Biol 49:557-583

Del-Campo JA, Rodríguez H, Moreno J, Vargas MÁ, Rivas J, Guerrero MG (2004) Accumulation of astaxanthin and lutein in Chlorella zofingiensis (Chlorophyta) Appl Microbio Biotechnol 64:848-854

Donkin P (1976) Ketocarotenoid biosynthesis by Haematococcus lacustrus. Phytochem 15:711718

Fraser PD, Miura Y, Misawa N (1997) In vitro characterization of astaxanthin biosynthetic enzymes. J Biol Chem 272:6128-6135 
Fraser PD, Shimada H, Misawa N (1998) Enzymic confirmation of reactions involved in routes to astaxanthin formation, elucidated using a direct substrate in vitro assay. Eur $\mathrm{J}$ Biochem $252: 229-236$

Grung M, D’Souza FML, Borowitzka M, Liaaen-Jensen S (1992) Haematococcus pluvialis aplanospores as a source of (3S, 3'S)-astaxanthin esters. J Appl Phycol 4:165-171

Guerin M, Huntley ME, Olaizola M (2003) haematococcus astaxanthin: applications for human health and nutrition. Trends Biotechnol 21:210-216

Ip PF, Chen F (2005) Production of astaxanthin by the green microalga Chlorella zofingiensis in the dark. Process Biochem 40:733-738

Ip PF, Wong KH, Chen F (2004) Enhanced production of astaxanthin by the green microalga Chlorella zofingiensis in mixotrophic culture. Process Biochem 39:1761-1766

Johnson EA, An G-H (1991) Astaxanthin from microbial sources. Crit Rev Biotechnol 11:297326

Johnson EA, Schroeder WA (1995) Microbial carotenoids. Adv Biochem Eng Biotechnol $53: 119-178$

Kajiwara S, Kakizono T, Saito T, Kondo K, Ohtani T, Nishio N, Nagai S, Misawa N (1995) Isolation and functional identification of a novel cDNA for astaxanthin biosynthesis from Haematococcus pluvialis, and astaxanthin synthesis in Escherichia coli. Plant Mol Biol 29:343-552

Kobayashi M, Kakizono T, Nagai S (1993) Enhanced carotenoid biosynthesis by oxidative stress in acetate-induced cyst cells of a green unicellular alga, Haematococcus pluvialis. Appl Environ Microbiol 59:867-873 
Lee PC, Schmidt-Dannert C (2002) Metabolic engineering towards biotechnological production of carotenoids in microorganisms. Appl Microbiol Biotechnol 60:1-11

Linden H (1999) Carotenoid hydroxylase from Haematococcus pluvialis: cDNA sequence, regulation and functional complementation. Biochem Biophys Acta 1446:203-211

Lorenz RT, Cysewski GR (2000) Commercial potential for Haematococcus microalgae as a natural source of astaxanthin. Trends biotechnol 18:160-167

Lotan T, Hirschberg J (1995) Cloning and expression in Escherichia coli of the gene encoding $\beta$ C-4-oxygenase, that converts $\beta$-carotene to the ketocarotenoid canthaxanthin in Haematococcus pluvialis. FEBS Lett 364:125-128

Margalith PZ (1999) Production of ketocarotenoids by microalgae. Appl Microbiol Biotechnol $51: 431-438$

McCarthy SS, Kobayashi MC, Niyogi KK (2004) White mutants of Chlamydomonas reinhardtii are defective in phytoene synthase. Genetics 168:1249-1257

Misawa N, Satomi Y, Kondo K., Yokoyama A, Kajiwara S, Saito T, Ohtani T, Miki W (1995) Structure and functional analysis of a marine bacterial carotenoid biosynthesis gene cluster and astaxanthin biosynthetic pathway proposed at the gene level. J Bacteriol 177:6575-6584

Orosa M, Valero JF, Herrero C, Abalde J (2000) Comparison of the accumulation of astaxanthin in Haematococcus pluvialis and other microalgae under $\mathrm{N}$-starvation and high light conditions. Biotechnol Lett 23:1079-1085

Rise M, Cohen E, Vishkautsan M, Cojocaru M, Gottlieb HE, Arad S (1994) Pigment and structural changes in Chlorella zofingiensis upon light and nitrogen stress. J plant Physiol $144: 287-292$ 
Sambrook J, Fritsch EF, Maniatis T (1989) Molecular Cloning: A Laboratory Manual, $2^{\text {nd }}$ ed., Cold Spring Harbor Laboratory Press, Cold Spring Harbor, NY.

Sandmann G (1994) Carotenoid biosynthesis in microorganisms and plants. Eur J Biochem $223: 7-24$

Shi XM, Liu HJ, Zhang XW, Chen F (1999) Production of biomass and lutein by Chlorella protothecoides at various glucose concentrations in heterotrophic cultures. Process Biochem $34: 341-347$

Stadler R, Wolf K, Hilgarth C, Tanner W, Sauer N (1995) Subcellular localization of the inducible Chlorella HUP1 monosaccharide- $\mathrm{H}^{+}$symporter and cloning of a co-induced galactose- $\mathrm{H}^{+}$symporter. Plant Physiol 107:33-41

Steiger S, Sandmann G (2004) Cloning of two carotenoid ketolase genes from Nostoc punctiforme for the heterologous production of canthaxanthin and astaxanthin. Biotechnol Lett 26:813-817

Stewart CN, Via LE (1993) A rapid CTAB isolation technique useful for rapid fingerprinting and other PCR application. Biotechn 14:748-751

Tanner W (1969) Light-driven uptake of 3-O-methylglucose via an inducible hexose uptake system of Chlorella. Biochem Biophys Res Commun 36:278-283

Wolf K, Tanner W, Sauer N (1991) The Chlorella $\mathrm{H}^{+} /$hexose cotransporter gene. Curr Genet $19: 215-219$ 


\section{Figure legends}

Fig. 1. Proposed pathway of astaxanthin biosynthesis in algae. Solid arrows represent documented reactions and dash arrows indicate speculated reactions in the biosynthesis pathway.

Fig. 2. PCR amplification of C. zofingiensis bkt gene. Lane 1: nested PCR product with primers dbktf2 + dbktr2; lane 2: product of 5'RACE; lane 3: product of 3'RACE; lane 4: RT-PCR product of full-length $b k t$ cDNA; M: 100 bp DNA ladder plus (Fermentas).

Fig. 3. Southern analysis of genomic DNA from C. zofingiensis. Genomic DNA (8 $\mu$ g) was digested with KpnI (lane 2), BamHI + EcoRI (lane 3), or PstI + XbaI (lane 4) respectively, electrophoretically separated on a $0.8 \%$ agarose gel, blotted onto a nylon membrane, and hybridized with a 928-bp bkt gene fragment amplified by PCR. Plasmids (400 pg) containing the bkt gene was cut by SacI and used as a positive control (lane 1).

Fig. 4. HPLC chromatogram of carotenoid pigments extracted from E. coli cells carrying plasmid pACCAR16 $\Delta \mathrm{crtX}$ and pCzb (A), pACCAR16 $\Delta \mathrm{crtX}(\mathrm{B}), \mathrm{pACCAR} 25 \Delta \mathrm{crtX}$ and pCzb (C), or pACCAR25 $\Delta \mathrm{crtX}$ (D). Peaks were monitored at $480 \mathrm{~nm}$ and were identified as follows: 1canthaxanthin; 2-echinenone; 3- $\beta$-carotene; 4- astaxanthin; 5-adonixanthin; 6- zeaxanthin.

Fig. 5. Analysis of the differential expression of bkt gene in C. zofingiensis cells using RT-PCR. RT-PCR was performed using RNAs from cells grown in a medium without glucose for $6 \mathrm{~h}$ (lane 1), $48 \mathrm{~h}$ (lane 7), and with 2\% glucose for $6 \mathrm{~h}$ (lane 2), $12 \mathrm{~h}$ (lane 3), $24 \mathrm{~h}$ (lane 4), $36 \mathrm{~h}$ (lane 5), and 48 h (lane 6); M: 100 bp DNA ladder plus (Fermentas). The 423-bp bkt specific PCR 
product was separated on a $1.2 \%$ agarose gel (A), along with internal controls (actin) amplification (B). 
Fig. 1

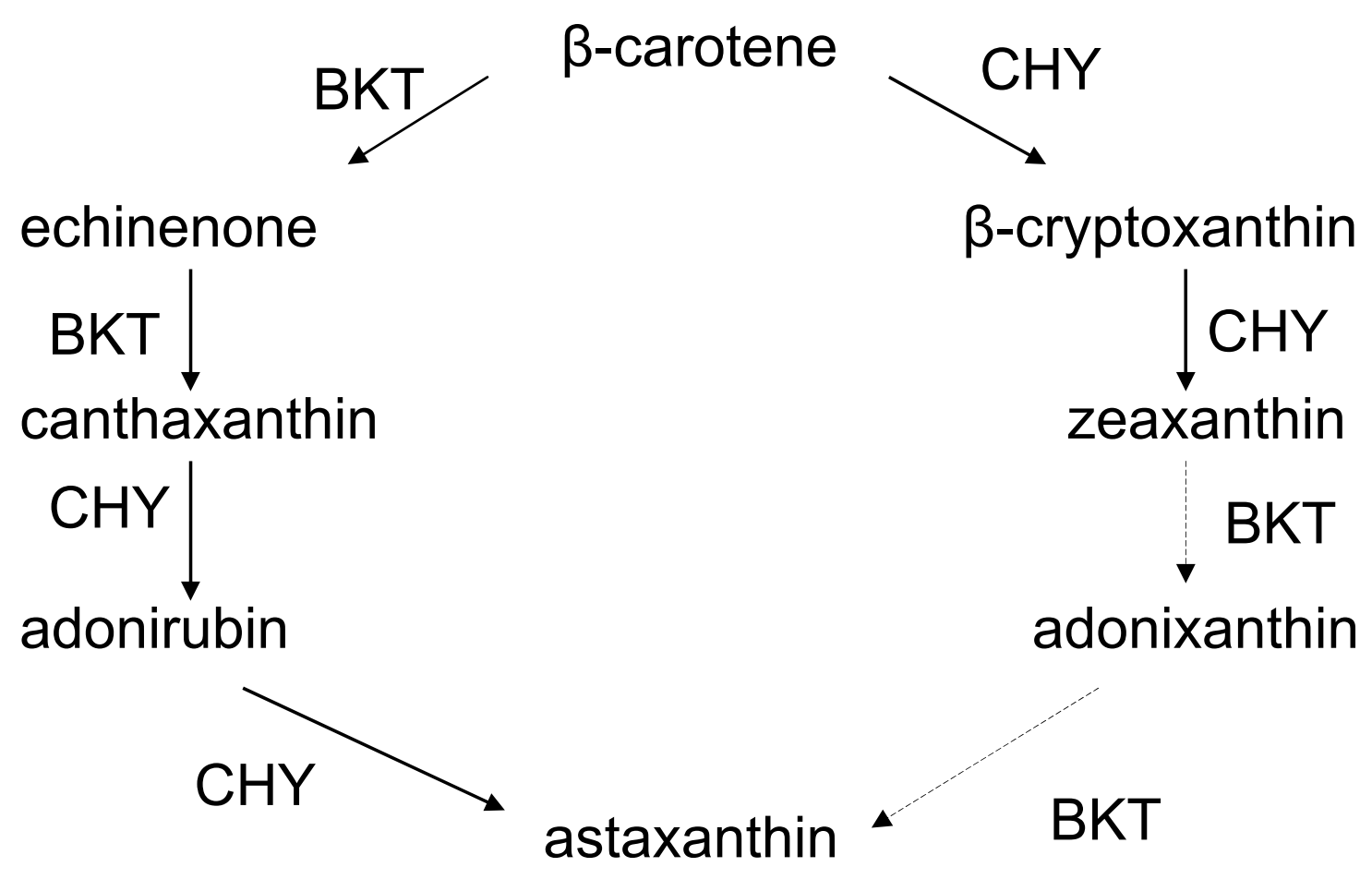


Fig. 2

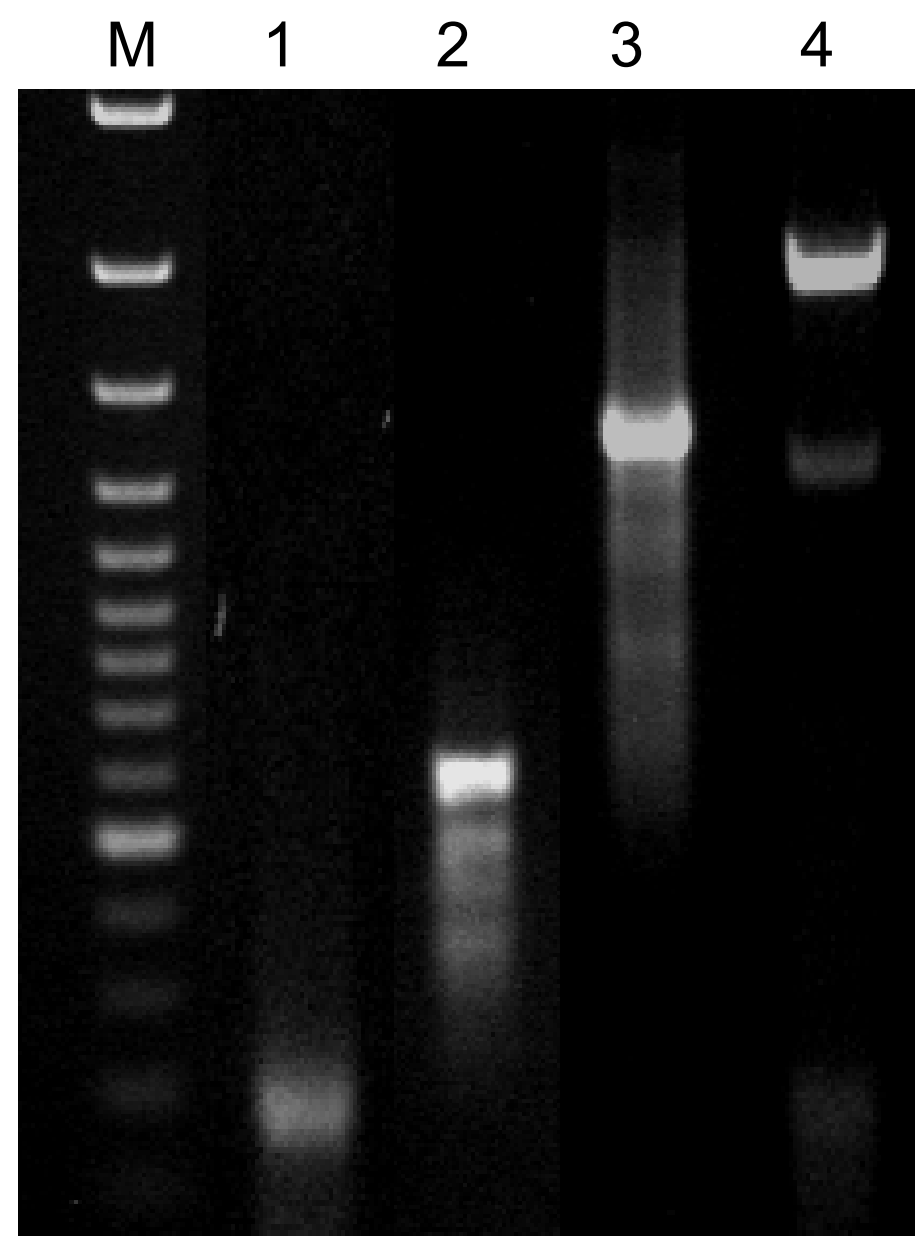


Fig. 3

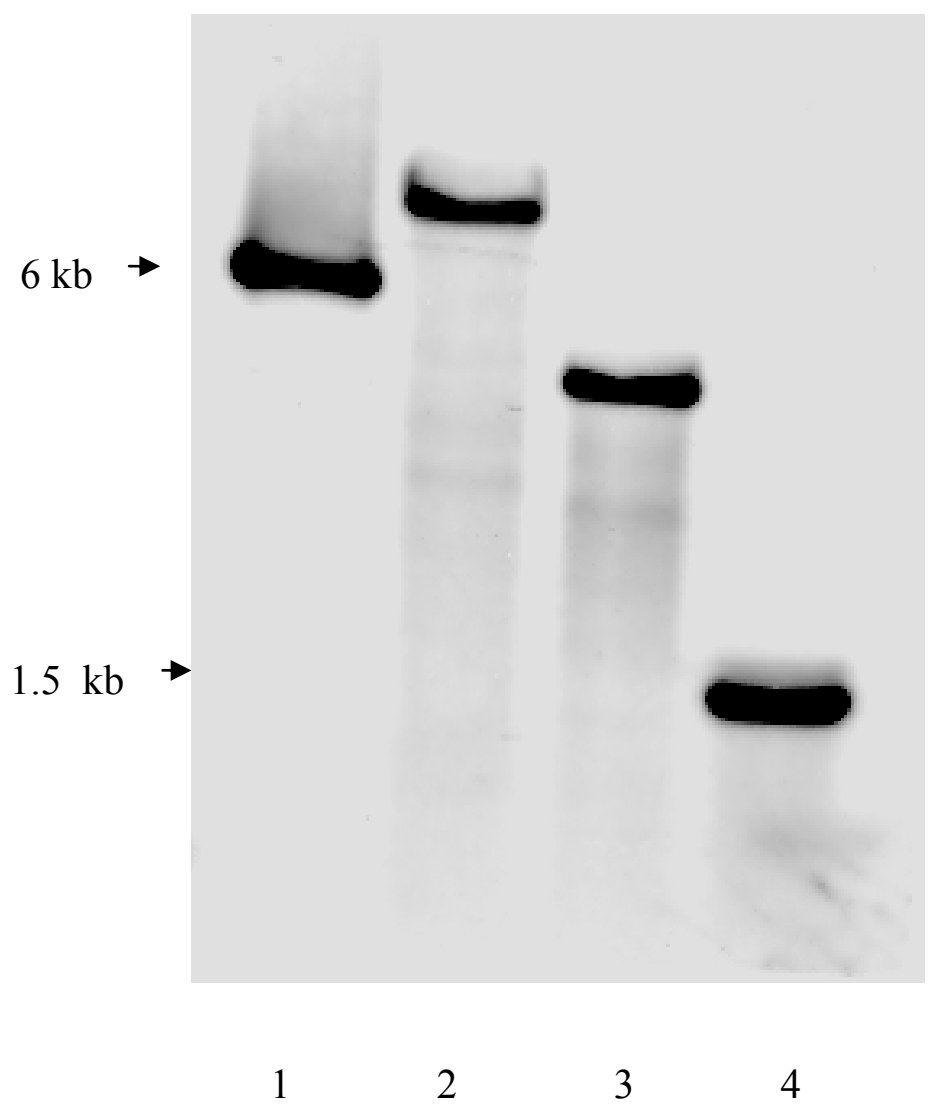


Fig. 4
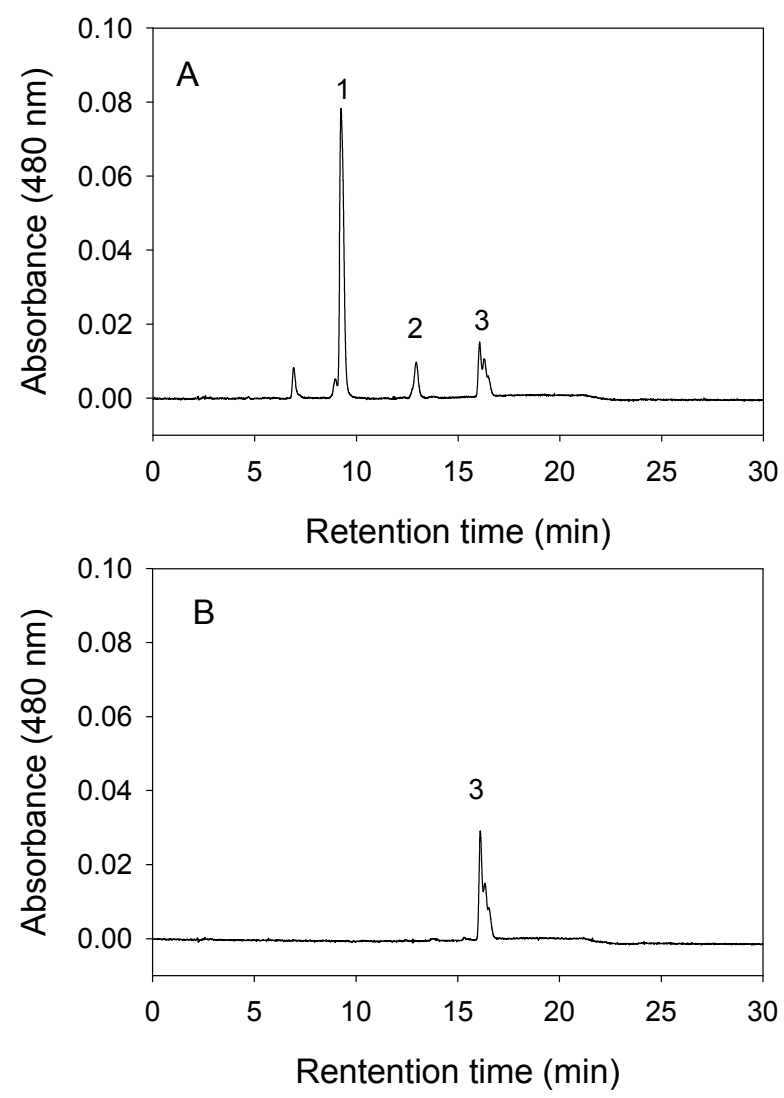
Fig. 4
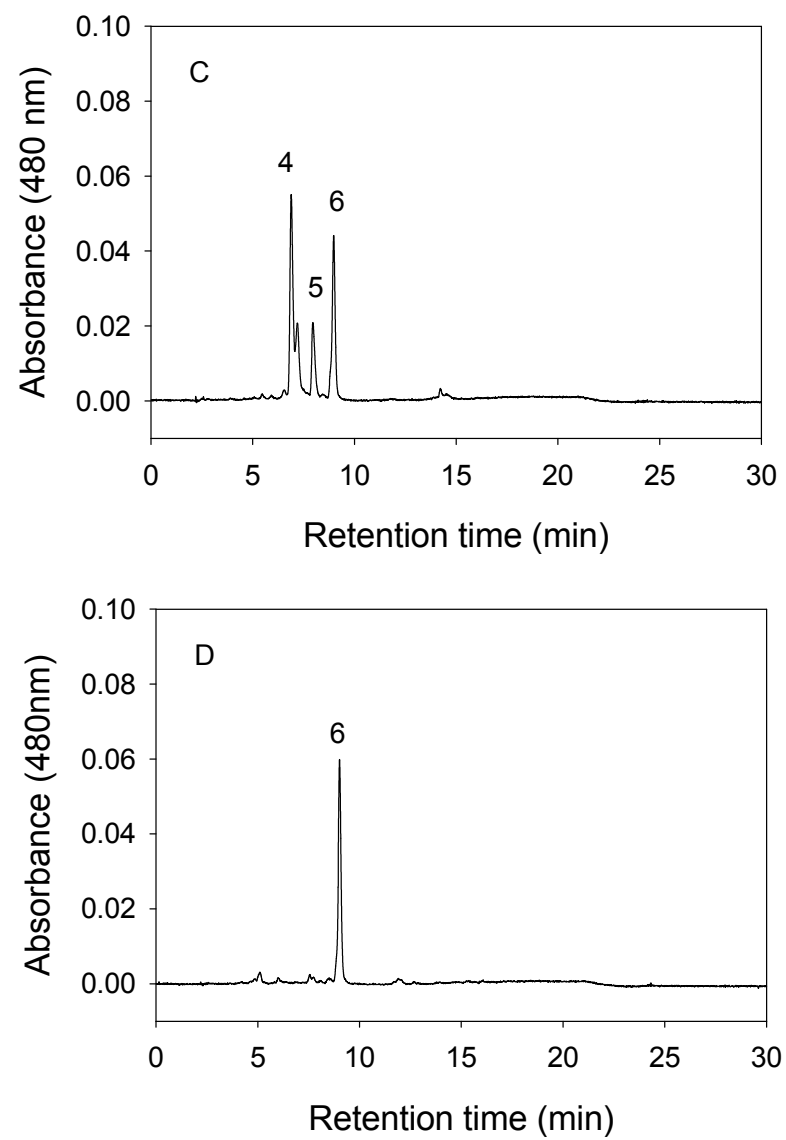
Fig. 5

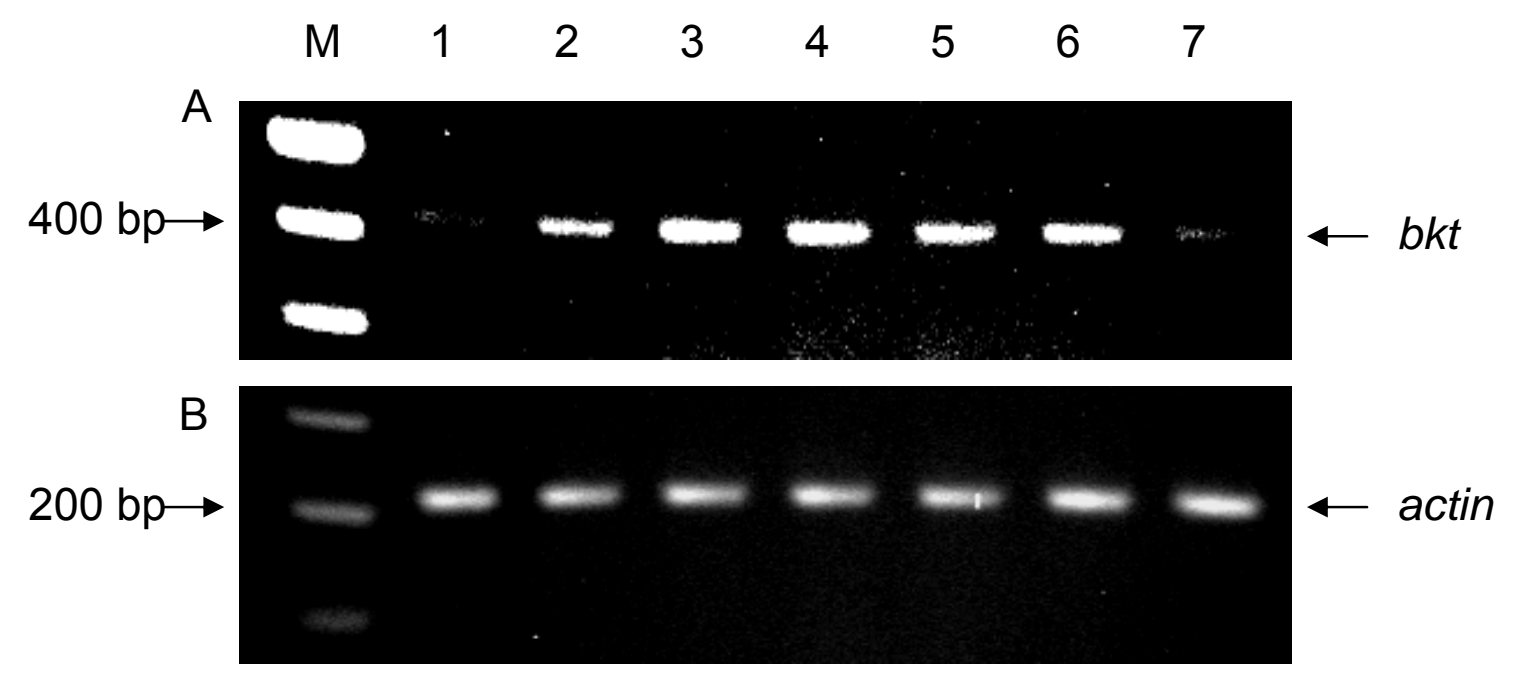

